\title{
Improved estimation of discard mortality rates with in situ experiments involving electronic and traditional tagging
}

\author{
Benoît Hugues P. ${ }^{1,{ }^{*}}$, Morfin Marie ${ }^{2}$, Capizzano Connor W. 3,4
}

${ }^{1}$ Fisheries and Oceans Canada, Maurice Lamontagne Institute, Mont Joli, QC, G5H 3Z4, Canada

2 IFREMER, Unité de Sciences et Technologies halieutiques, Laboratoire de Technologie et Biologie

Halieutique, F-56100, Lorient, France

${ }^{3}$ Anderson Cabot Center for Ocean Life, New England Aquarium, Boston, MA, 02125, USA

${ }^{4}$ School for the Environment, University of Massachusetts Boston, Boston, MA, 02125, USA

*Corresponding author : Hugues P. Benoît, email address : hugues.benoit@dfo-mpo.gc.ca

\begin{abstract}
:
Reliable estimates of release or discard mortality (DM) rates for recreational and commercial fisheries are necessary for robust assessment of the effects of fishing on populations and for establishing effective regulatory measures concerning the release of fish. In situ experiments involving the tagging of released fish are most likely to produce accurate estimates given that experimental subjects are captured and released under representative fishery conditions. Data from electronic tags can be used to infer movement or behavioral patterns of released fish, from which mortality times can be derived to then estimate natural mortality and DM rates. However, resulting DM rate estimates are often of low precision (high variance) due to small sample sizes associated with the elevated cost of electronic tags. Alternatively, returns of traditional tags from small scale experiments constitute a relatively inexpensive means of estimating the relative DM rates of two or more groups (e.g., vitality or injury classes, capture methods) of released fish. Combining the two approaches when there are two or more groups of discarded individuals can be beneficial. First, results from the two methods can be used to assess whether experimental effects such as tag-related mortality could bias estimates of DM rates. Second, we propose a modelling approach that integrates data from these two types of tagging methods to produce absolute DM rate estimates that can be more precise than those obtained from electronic tagging alone. We show using simulations under which conditions precision is improved and how different allocations of effort to electronic and traditional tagging can be used to plan DM experiments with respect to the precision of estimates and cost. An example based on a recent study is presented to illustrate the application of the proposed approach.
\end{abstract}

\section{Highlights}

- Discard mortality rate estimates are required for robust assessment and management. Traditional or electronic tags are typically used; both approaches have limitations. Combining data from the two source can improve the precision of estimates. Discard mortality experiments can be planned with respect to cost and precision. 
Keywords : Catch and release mortality, Electronic tagging, Capture-mark-recapture tagging, Parametric survival modelling, Integrated modelling 


\section{Introduction}

Demand for estimates of mortality rates associated with the release or discarding of fish captured in recreational or commercial fisheries (hereafter, discard mortality, DM) has increased in recent years. This has been motivated in part by the desire to set effective fishery management measures, such as size and possession limits, and mandatory release policies (e.g., Coggins et al., 2007; Cooke and Schramm, 2007; Cowx et al., 2010) or landings obligations (e.g., European Union Common Fisheries Policy, European Commission, 2013). In addition, this demand is motivated by a desire to more accurately account for dead discards in population assessments (Punt et al., 2006; Cook, 2019; Swain et al., 2019). Given that discarding can represent an important component of fishing-related mortality in both recreational and commercial fisheries, it is important that DM rate estimates be based on the best available science (Harrington et al., 2005; Coggins et al., 2007; Davies et al., 2009; Radford et al., 2018).

The quality of published DM rate estimates has been variable, owing to limitations of the experimental approaches employed and to some extent by the analytical approaches used to produce these estimates (Pollock and Pine, 2007; ICES, 2014, 2019). Experimental approaches can be separated into two categories, containment and tagging, based on the treatment of fish after they are captured using actual or simulated fishing. In containment studies, the fate of fish is monitored by confining them to on-deck or shore-based tanks (e.g., Benoît et al., 2012) or in situ enclosures (e.g., Sulikowski et al., 2018). The quality of resulting DM rate estimates may be low due to biases associated with confinement (e.g., additional stress, absence of predators) and the difficulty of obtaining true control subjects (Pollock and Pine, 2007; ICES, 2014). In contrast, many biases associated with containment studies are not applicable to tagging approaches because individuals are returned to their environment, where they are subject to natural sources of mortality, including predators (Raby et al., 2014; Capizzano et al., 2016). However, tagging studies are not without bias, as tag-related mortality can be confounded with DM.

Tagging studies for estimating DM rates can be sub-divided into two types, those based on the recapture of animals tagged with traditional tags and those that track the fate of individuals bearing electronic tags. Population scale DM rate estimates can be directly obtained in long-term traditional tagging studies involving multiple release periods with both control and experimental fish, particularly if reporting rates can be reliably estimated and if exploitation rates, and therefore the potential for tag returns, are high (Pollock and Pine, 2007; Polacheck et al., 2010). These constraints generally limit the traditional tagging approach to well monitored fisheries. In the absence of multiple release periods, and even absent reporting rate estimates, the relative DM rates between groups of tagged fish can be estimated, where groups can be defined on the basis of capture conditions or by degrees of pre-release injury or impairment (e.g., Kaimmer and Trumble, 1998; Hueter et al., 2006; Stachura et al., 2012). These estimates can only be treated as absolute estimates if the DM rate of one of the groups can be assumed to be known. Because this approach does not require an estimate of reporting rate, assuming only that it is the same between groups, experiments can be done at scales smaller than the population and likely at modest cost.

Data from electronic tags can be used to infer the fate of released fish based on movement patterns evident in telemetry data (e.g., Yergey et al., 2012; Capizzano et al., 2016; Kerns et al., 2016) or changes in environmental parameters recorded in pop-up archival tags (e.g., Domeier et al., 2003; Kerstetter et al., 2003; Campana et al., 2009). Resulting estimates of mortality times can then be used to estimate natural mortality and DM (Benoit et al., 2015; Hightower and Harris, 2017). While the electronic tagging approach does not specifically require the use of minimallyharmed control fish to estimate DM, it does require estimates of tag-related mortality and 
information to correctly classify fish as being dead based on patterns in the telemetry or environmental data. A principal weakness of this method is that the precision of DM rate estimates is often low (i.e., the variability of the estimates is high) due to small sample size associated with the elevated cost of the tags (e.g., Musyl et al., 2011a, 2014; Capizzano et al., 2016). Furthermore, compared to traditional tagging, electronic tags are perhaps more likely to result in tag-related mortality due to their relatively large size and the possibility of predator attraction (e.g., Stansbury et al., 2015), and disentangling this mortality from DM is not straightforward, particularly if the two interact (i.e., higher tagging mortality in impaired or injured fish).

Combining electronic and traditional tagging in experiments involving two or more groups of discarded individuals could be beneficial. First, results from the two methods could be used in some instances to identify possible biases in DM rate estimates resulting from electronic tagrelated mortality. If the groups of fish are based on a health-related indicator such as the degree of injury, condition or behavioural impairment, as is often the case (e.g., Kaimmer and Trumble, 1998; Hueter et al., 2006; Benoît et al., 2010), and if fish 'health' interacts with tag-related mortality as might be expected (e.g., the electronic tag further impairs feeding or predator avoidance, or attracts predators that certain groups have more difficulty evading), then relative group-specific mortality rates should differ between estimates from traditional versus electronic tagging. Comparing the results obtained from the two types of tagging can therefore flag this type of bias. The second potential benefit is the focus of this paper. We propose a modelling approach that integrates data from both electronic tagging and small scale traditional tagging of two or more groups of discarded fish to address some of the shortcomings of the respective methods. This is achieved by jointly evaluating the likelihood of the two sources of data in the same likelihood function (c.f., Maunder and Punt, 2013). The approach produces absolute DM rate estimates that can be more precise (smaller error) than those obtained from electronic tagging alone. We use simulations to show under which conditions precision is improved and to which degree, and to illustrate how the allocation of effort to electronic and traditional tagging could be used to plan DM experiments with respect to the precision of estimates and cost. We illustrate the application of the proposed approach in a brief case study based on recent experiments aimed at estimating DM rates for haddock released in a recreational fishery (Capizzano et al., 2019).

\section{Methods and Materials}

\subsection{The model}

The integrated analysis builds on the model for longitudinal (event) data proposed by Benoît et al. (2015). This model is appropriate for data obtained (quasi-)continuously using electronic tags. At its core is a mixture distribution model of survivorship over time, $S(t)$, for discarded fish that were alive at the time of release:

$$
S(t)=\pi \exp \left[-(\alpha t)^{\gamma}\right]+(1-\pi)
$$

where $\alpha$ and $\gamma$ are parameters of the Weibull survival function that describes the attrition of fish that will die after release, and $\pi$ is the proportion of fish that were affected by capture and handling and will eventually die as a result (i.e., the post-release mortality rate), whereas $1-\pi$ is the unaffected proportion. A recent meta-analysis of longitudinal DM studies found that values of $\alpha$ and $\gamma$ are such that affected individuals typically die within days, rather than weeks or months $(\mathrm{H}$. Benoît and M. Morfin, unpublished analyses).

Benoît et al. (2015) further generalized eq. 1 to include natural mortality $(M)$ and capture and handling mortality $(\mathrm{CH})$ : 


$$
S(t)=\left(\pi \exp \left[-(\alpha t)^{\gamma}\right]+(1-\pi)\right) \cdot \tau \cdot \exp (-M t)
$$

where $1-\tau$ is the $\mathrm{CH}$ rate, $\tau \pi$ is the post-release mortality (R) rate, $1-\tau+\tau \pi$ is the total capture-handling-release (CHR) process mortality rate and $M$ is the instantaneous rate of natural mortality (for a derivation see Benoît et al., 2015). Hereafter, we use CHR to refer to the process and DM rate to refer to the mortality rate that results from it.

In this model $\alpha, \gamma, \pi, \tau$ and $M$ are all estimated (and estimable) parameters. The model is able to reliably distinguish DM and $M$ under many scenarios because the two sources of mortality act over different time scales (much shorter for DM) and because the CHR component of mortality converges to the asymptote $\tau(1-\pi)$ (Benoit et al., 2015). Contrary to other approaches (e.g., Kerns et al., 2016; Hightower and Harris, 2017), there is no requirement for the analyst to arbitrarily choose a time when DM is believed to have been fully expressed such that remaining mortality can be attributed to $M$ or fishing mortality (removals).

The survivorship of two or more groups of fish can be modelled in eq. 2 by adding a group effect to one or more of the model parameters. In the case of the parameters that affect DM, $\pi$ and $\tau$, the effect of group (or other covariates such as environmental conditions) can be modelled in a linear predictor that is related to these parameters using a logit link to ensure that they remain bounded over the interval $[0,1]$ (Benoît et al., 2012, 2015) :

$$
\begin{gathered}
\operatorname{logit}\left(\tau_{g}\right)=X \beta_{1} \\
\operatorname{logit}\left(\pi_{g}\right)=X \beta_{2}
\end{gathered}
$$

in which $X, \beta_{1}$ and $\beta_{2}$ are respectively the design matrix and the vectors of parameters for the effect of group on $\tau$ and $\pi$.

Estimates for the parameters of eqs. 2-4, can be obtained by maximum likelihood conditionally on the observations from each individual $i$ from group $g$ of the variable $Y_{g, i}$, which is either its mortality time $T_{g, i}$ (uncensored data), or an upper or lower bound of $T_{g, i}$ (left-censored or rightcensored data respectively), as well as the type of censoring. The variables $T_{g, i}$ are assumed independent and distributed according to the survival distribution function, $S(t) g$, i.e. the probability that the individual survives to a time $T_{g, i}>t$, as defined in eqs. 2-4.

For uncensored data, the likelihood function given the event time $y_{g, i}$ from group $g$ is the probability density function of $T_{g, i}$, which is the first-derivative of $1-S(t)_{g}$ (for the derivation see Benoît et al., 2015; Ibrahim et al., 2001):

$$
m(t)_{g}=\left(\pi_{g} \cdot\left(\alpha \gamma \cdot(\alpha t)^{\gamma-1}+M\right) \cdot \tau_{g} \cdot \exp \left(-[\alpha t]^{\gamma}\right)+\left(1-\pi_{g}\right) \cdot M \cdot \tau_{g}\right) \cdot \exp (-M t)
$$

If censoring processes are independent of the variables $T_{g, i}$, the likelihood function for both right and left-censored observations are a simple function of the survival distribution function in eq. 2 (see eq. 6 below).

Now, let $y$ denote the set of independent observed event times (mortality or censorship, as the case may be) spread across $G$ groups of fish, with $b_{g}$ the number of fish in each group. The resulting full log-likelihood function for the mixture distribution model for the electronic tagging data is given by:

$$
\begin{gathered}
l(\Theta \mid y, d, e)_{\text {electronic }}=\sum_{g=1}^{G} \sum_{i=1}^{b_{g}}\left(1-e_{g, i}\right)(1- \\
\left.d_{g, i}\right) \log \left(m\left(y_{g, i}\right)_{g}\right)+e_{g, i} \log \left(S\left(y_{g, i}\right)_{g}\right)+d_{g, i} \log \left(1-S\left(y_{g, i}\right)_{g}\right)
\end{gathered}
$$


where $\Theta$ denotes the vector of unknown parameters $\left(\alpha, \gamma, \beta_{1}, \beta_{2}, M\right), d$ is a vector for a binary variable that denotes whether observations are left-censored $(d=1)$ or not $(d=0)$, and $e$ is a vector for a binary variable denoting whether observations are right-censored. The treatment of leftcensored observations is important in estimating the $\mathrm{CH}$ mortality reflected in $\tau_{g}$.

Now suppose that as part of the same experiment, some fish classified into each group were individually tagged with traditional tags and released. We assume that there are no differences between groups in terms of subsequent catchability, fishing effort, and tag-return rates, which are all factors that jointly affect the likelihood of recovering tags in addition to survival differences. Based on these assumptions, the probabilities for each group, $p_{t g}$, that a tag recovered at time $t$ and returned belongs to that group is simply a function of the relative survivorship functions for each group:

$$
p_{t}=\left(p_{t 1}, \ldots, p_{t G}\right)=\left(\frac{n_{1} S(t)_{1}}{\sum_{g=1}^{G} n_{g} S(t)_{g}}, \ldots, \frac{n_{G} S(t)_{G}}{\sum_{g=1}^{G} n_{g} S(t)_{g}}\right) \text { where } \sum_{g=1}^{G} p_{t g}=1
$$

and where $n_{g}$ is the number of fish tagged and released in group $g$ and $S(t)_{g}$ is the complete mixturedistribution survivorship function (eq. 2) for an individual in group $g$. Given the number of tags returned for each group and time of recapture, $r_{t g}$, a multinomial distribution can be used to evaluate the log-likelihood function of the tag-return data:

$$
l(p \mid r)_{\text {tag return }}=\sum_{t=0}^{t_{\max }}\left(C_{t}+\sum_{g=1}^{G} r_{t g} \log p_{t g}\right)
$$

where $C_{t}$ is the multinomial coefficient for the data at time $t$.

The assumptions of equal catchability, fishing effort, and tag-return rates among groups are likely to be true provided that the behaviour of fish released does not differ among groups or that any differences are constrained to a recovery period that is short relative to the period over which tags are recovered (Hueter et al., 2006). Effects of CHR that cause certain groups to be less active for prolonged periods following release could reduce catchability and may limit dispersal movements that in turn affect the local fishing effort directed at them (thus opportunities for recapture) relative to other groups. This may occur if groups are based on degrees of injury or impairment resulting from CHR, although many studies employing such groups implicitly or explicitly considered the above assumptions to be verified (e.g., Kaimmer and Trumble, 1998; Hueter et al., 2006; Stachura et al., 2012; Yochum et al., 2018). Data from electronic tagging can be used to evaluate whether behavioural differences exist between groups of fish and whether they are constrained to a short recovery period for which any associated traditional tag returns could be omitted from the analysis. Evidence from several electronic tagging studies suggests that behavioural changes following release tend to be of short duration (hours to days) and most common in fish that eventually die purportedly as a result of CHR (Campana et al., 2009; Capizzano et al., 2016, 2019). (Note that it is still possible to estimate the relative survival of groups under the less restrictive assumption of constant relative catchability, effort, and return rates between groups, but only if their relative instantaneous survival rates diverge over time (Hueter et al., 2006), something that is inconsistent with the asymptotic nature of DM in eq 2).

Assuming that the survivorship of fish affixed with electronic or traditional tags is dictated by the same CHR process and natural mortality, the two sets of data carry information about the parameters that affect that survivorship. While the electronic tagging data can be used to inform values for all parameters of eq 2 , including parameters in the linear predictors that define how the main model parameters differ between groups (eqs 3-4), the tag return data can only inform the 
latter. Because the data sets are independent, their respective log-likelihoods can simply be added to yield a single likelihood function to estimate parameters as part of an integrated analysis:

$$
l(\Theta \mid y, d, e, r)=l(\Theta \mid y, d, e)_{\text {electronic }}+l(p \mid r)_{\text {tag return }}
$$

The assumption above will not be true if there is experimentally-induced mortality that differs between the two types of tagging, such as higher tagging-related mortality for electronic tags. If this mortality is strictly additive to DM in that its magnitude does not differ among groups, the approach in eq. 9 remains valid in that relative rates of mortality will not be affected, although resulting DM estimates will be biased by the experimental effect. In contrast, the approach in eq. 9 will not be valid if the experimentally-induced mortality interacts with group-specific DM, such as would occur if groups comprising healthier/uninjured fish are more resilient to mortality caused by electronic tagging. Such an effect is fundamentally a flaw in the experimental approach that leads to biased estimates of group-specific DM, not a failing or limitation of the approach proposed here.

\subsection{Simulations}

We used simulations to evaluate how the precision and possible bias of DM rate estimates vary as a function of the number of electronic tags deployed and traditional tags returned assuming the assumptions for eq 9 are satisfied. We considered effects on both group-specific estimated DM rates and on an overall average DM rate obtained as a weighted average of group-specific rates. The latter approach has been used to scale up the results of DM experiments to entire fisheries by estimating the frequency distribution of group membership in the fishery (e.g., the average number of fish in each injury-level group) and using these values as weights to calculate an average DM rate (Benoît et al., 2012, 2015; Capizzano et al., 2016; Sulikowski et al., 2018).

For simplicity we independently simulated the survivorship of either two, three or four groups of fish using eq 2., but with no $\mathrm{CH}$ mortality $(\tau=1)$ and assuming that survival differences were only reflected in the parameter $\pi$ :

$$
S(t)_{g}=\left(\pi_{g} \exp \left[-(\alpha t)^{\gamma}\right]+\left(1-\pi_{g}\right)\right) \cdot \exp (-M t)
$$

In all simulations, all parameters except $\pi_{g}$ were kept fixed at values consistent with previous studies and because the results of these simulations should be insensitive to reasonable alternative values (Benoît et al., 2015): $\alpha=0.22 ; \gamma=1.99 ; M=5.48 \mathrm{e}^{-4}$, which is equivalent to an annual rate of 0.2 . Each set of simulations differed based on the simulated vector for $\pi$. There were five sets of simulations based on two groups, $\left(\pi_{1}=0.75, \pi_{2}=0.70\right),(0.75,0.50),(0.75,0.25),(0.25,0.50)$, and $(0.25,0.30)$, three sets based on three groups, $(0.75,0.70,0.50),(0.75,0.50,0.25)$, and $(0.50,0.30$, $0.25)$, and one set based on four groups $(0.75,0.70,0.50,0.25)$. Jointly these sets were used to evaluate the influence of the DM rate magnitude, the relative difference in DM rates and the number of groups employed on the precision and bias of DM rate estimates, as a function of the number of electronic and traditional tags. Within each set, we simulated studies comprising from zero to 200 tag returns and from 40 to 400 electronic tag deployments. In all cases we assumed that an equal number of fish were tagged and released in each group.

The simulation process was iterative, whereby each iteration consisted of simulating from the model in eq 10 and estimating the parameters assuming that the model is correct, since it was not our objective to evaluate the consequences of model misspecification. The estimation involved minimizing the negative of the log-likelihood in eq 9. 
The model simulations were based on those of Benoît et al. (2015) and are a modification of the generalized method of Bender et al. (2005). One-thousand iterations were sufficient to provide sufficiently stable results in all cases. During each iteration and for each simulated individual, $i$, a value of $S(t)_{i}$ was drawn from a uniform distribution:

$$
S(t)_{i} \sim U(0,1)
$$

The associated survival time $T_{i}$ was then obtained by solving eq 10 using the Newton-Raphson algorithm. For each simulated electronically-tagged individual, a censoring time was also simulated, and the lesser of the survival and censoring times was used as the simulated observation for that individual. Following Benoît et al. (2015), censoring times were drawn from a modified Beta distribution ( $\alpha=0.7, \beta=1.4$ and with support rescaled to 185 days). (Note that those authors found that simulation results were insensitive to reasonable alternative censoring functions).

Tag returns were simulated by first simulating the survival times for 3,000 fish, again assuming an equal split of initial tagging between groups. Tag recovery times were drawn randomly from a uniform distribution of times ranging between 0 and 365 days. Results were insensitive to other functions for recovery times, including temporally clustered times (not shown). For each recovery time, a fish was sampled without replacement from among those alive at that time; effectively sampling with probabilities equal to the relative number of live fish in each group. This procedure did not simulate stochasticity that would be associated with small release numbers.

For each set of simulations we calculated the following metrics:

1) the mean percent bias of group-specific DM rate estimate, $P B_{g}=\frac{100 \sum_{i=1}^{1000}\left(\widehat{\pi}_{g i}-\pi_{g} \text { true }\right)}{1000}$ ;

2) the coverage rate for $95 \%$ confidence intervals for group-specific DM rate estimates, estimated using the standard errors estimated when fitting the model to the data simulated in each iteration;

3) the coefficient of variation (CV) of group-specific DM rate estimates; and,

4) the pairwise correlations between group-specific DM rate estimates.

In addition, for each simulation set we also calculated the CV (across iterations) for an overall (weighted) group-aggregated average DM rate. For the simulation sets based on two groups we examined results for three cases of group-specific relative weights: $\left(w_{\text {group }}=0.5, w_{\text {group }}=0.5\right),(0.7$, 0.3 ) and $(0.9,0.1)$, where group $=2$ is the group with the smallest DM rate. For the remaining simulation sets we examined results for two cases, one with equal weights among groups, and another with a relative weight of 0.1 for the group with the smallest DM rate and equal relative weights for the remaining groups. The different cases were undertaken to illustrate how changes in the precision of group-specific DM rate estimates and the correlation in estimates among groups affect the precision of overall group-aggregated estimates.

Model fitting via maximum-likelihood and simulations were all undertaken using the $\mathrm{R}$ statistical computing software (R Core Team, 2018). Optimization of the log-likelihood functions was undertaken using the R optim function.

\subsection{Case study}

Capizzano et al. (2019) investigated the mortality of haddock (Melanogrammus aeglefinus) discarded in the recreational rod-and-reel fishery that occurs on Jeffrey's Ledge in the western Gulf of Maine (U.S.A., NW Atlantic). Briefly, 154 haddock were captured, sampled for a series of biological characteristics and externally tagged with an electronic tag, specifically a pressuresensing acoustic transmitter, before being released. The release area was continuously monitored 
using an array of 32 acoustic receivers that were spaced approximately $1.1 \mathrm{~km}$ apart, covering roughly $22 \mathrm{~km}^{2}$. The acoustic tags emitted a unique, coded signal and depth measurement every 2 min for the first $7 \mathrm{~d}$, every $5 \mathrm{~min}$ for the next $23 \mathrm{~d}$, and then every $15 \mathrm{~min}$ until transmissions terminated at $365 \mathrm{~d}$. Fish that exhibited minor vertical and horizontal movements similar to known dead tagged reference fish were considered dead, and the time of onset of those behaviors was taken as the time of death. The other living fish were monitored until they left the array, at which point they were considered right-censored observations. In addition to releasing fish with electronic tags, the authors also released 2,428 fish with traditional tags, of which 111 were recaptured and their tags returned. No efforts were made by the authors to solicit tag returns, such as signage at wharves or communications to fisher groups, although small value rewards (approximately \$10 US) were provided for tag returns. In the absence of a method to integrate the results of acoustic and traditional tagging such as proposed in the present paper, Capizzano et al. (2019) based their estimates of DM rate on the acoustic tagging results only.

One of the initial objectives of Capizzano et al. (2019) was to estimate the DM rate for haddock above and below a retention size limit of $43.2 \mathrm{~cm}$ (17 inches). Because their experiments were conducted at two times of the year, spring (April and May) and autumn (September), an effect of season was also considered. Here, we estimate the DM rate only of haddock above the size limit as a function of season, the sample sizes for which are rather modest (Table 1). There were too few tag returns for fish below the size limit to illustrate the benefit of including tag return data in DM rate estimates for these fish (19 returns of 1,077 tagged fish). Furthermore, concerns for the effects of size-dependent catchability on tag returns prevented us from producing estimates for all sizes combined.

Our example is only meant to be illustrative. We assumed that the potential to recover tags, irrespective of DM rate, was the same for the two seasons because the time interval between tagging events was short relative to the duration of the recreational fishing season. The validity of this assumption requires a more rigorous evaluation before using the results more formally.

\section{Results}

\subsection{Simulations}

In all sets of simulations, the estimates for parameters that were not simulated as group-specific $(\alpha, \gamma$ and $M)$ were not affected by the inclusion of tag-return data. This was expected because those data do not provide information on the value of those parameters and results are therefore not presented.

Estimated DM parameters were unbiased in all simulated cases with average percent bias values that varied randomly among cases between $-0.54 \%$ and $0.82 \%$, with an overall mean of $0.06 \%$. The coverage of confidence intervals was excellent in all simulated cases, with values that varied randomly among cases between 0.93 and 0.98 , with an overall mean of 0.952 . Thus, estimates of DM using the approach proposed here will be unbiased and will have correctly estimated error provided that assumptions outlined in the Methods and Material section are met.

We focus hereafter on describing results for the metrics that varied among simulated cases, the $\mathrm{CV}$ of DM estimates and the correlation in DM estimates between groups. Those results are presented using contour plots for the given metric, as a function of the number of simulated electronic tags (x-axes) and the number of simulated traditional tag returns (y-axes) (e.g., Fig. 1). In all simulated cases, the metrics were most sensitive to changes in the number of simulated traditional tag returns when the simulated number of electronic tags was small. Consequently a $\log$-scale was used for the $\mathrm{x}$-axes. 


\subsubsection{Simulations involving two groups}

The inclusion of tag return data had two principal effects on the estimation of group-specific DM rates. First, for a given number of electronic tags, increases in the number of traditional tag returns decreased the CV (i.e., indicating increased precision) of group-specific DM rates, particularly for the group with the largest DM rate (group 1 in Fig. 1a, group 2 Fig. 1b). For that group, the reduction in $\mathrm{CV}$ with the addition of returned tags was greatest when the number of electronic tags was small, as evidenced by the less steeply inclined contours in the panels. (Vertical contours indicate no reduction in $\mathrm{CV}$ with the addition of traditional tag returns). Moreover, reductions in $\mathrm{CV}$ for a given number of electronic tags were also a positive function of the difference in DM rates between groups within pairs (compare rows in Figs. 1a and in 1b). For instance, compared to including no tag returns at all, including 100 tag returns in simulations with 50 electronic tags and a group-one DM rate of 0.75 reduced the $\mathrm{CV}$ for the DM rate estimate for that group by $40 \%, 36 \%$ and $28 \%$, when group two had a DM rate of $0.25,0.50$ and 0.70 respectively. In all cases, reductions in $\mathrm{CV}$ with the addition of tag returns were much smaller for the group with the lower DM rate, except for the pair with group-specific rates of 0.75 and 0.70 (Fig. 1 top row).

The second effect of adding tag returns was to increase the correlation between estimated group-specific DM rates (Fig. 1, right column). The correlations increased as the number of tag returns increased for a given number of electronic tags. The rate of increase in the correlations was an inverse function of the number of electronic tags, the magnitude of the smallest group-specific DM rate in the pair, and, to a lesser extent, the difference in DM rate between the groups (compare rows in Figs. 1a and 1b). For instance, correlation values were modest across a range of electronic tag and traditional tag return numbers for the pair comprising group-specific DM rates of 0.75 and 0.25 , and slightly higher when there were fewer electronic tags for the pair comprising rates of 0.30 and 0.25 . In contrast, correlation values increased rapidly and to high levels (e.g., 0.8 ) for the pair comprising rates of 0.75 and 0.70 . This pattern explains the reductions in CV of the DM rate estimate for the latter group (Figure 1, top-centre panel).

Patterns in the CV of the overall average DM rate with respect to the number of electronic tags and traditional tag returns varied as a function of the weights applied to the two groups (Fig. 2 columns) and, to a lesser extent, the difference in simulated DM rates between the two groups (Fig. 2 rows). When the weights were equal for the two groups (Fig. 2 left column), the addition of tag returns for a given number of electronic tags only reduced the $\mathrm{CV}$ of the estimate for the pair comprising rates of 0.75 and 0.25 . This pair was also associated with the lowest correlations between estimates for a given number of each type of tag (Fig. 1). In contrast, the reduction in CV associated with the addition of tag returns increased in magnitude as the relative weights for the group associated with the smallest group-specific DM rate were reduced (Fig. 2 columns, from left to right), and as the within pair difference in simulated DM rates increased (rows, from top to bottom in Fig. 2a and bottom to top in Fig. 2b).

\subsubsection{Simulations involving three or four groups}

The general patterns observed when there were either three or four groups greatly resembled the results obtained for two groups. The rate of reduction in the CV of group-specific DM rate estimates with the addition of tag returns varied mainly as a function of the group-specific DM rate (diagonal panels in Figs. 3 to 6), and to a lesser degree as a function of the DM rates for other groups included in the same estimation (e.g., compare CV contour plots for a DM rate of 0.50 in Figs. 3, 4 and 5). The CV for a given group and numbers of both types of tags typically increased 
with the addition of a third and fourth group, a direct result of the smaller number of tags in each group (i.e., fixed total number of tags, split among more groups).

The rate of decrease in $\mathrm{CV}$ with the addition of tag returns increased as the number of groups increased from two to four for a given simulated group-specific DM rate. For example, for the group with a simulated DM rate of 0.75 , the contours are increasingly angled away from vertical when comparing the upper-left panel for Figs 1 (two groups), 3 or 4 (three groups), and then 6 (four groups). Furthermore, the correlation between estimated DM rates for a common pair of simulated DM rates, and a fixed number of electronic tags and tag returns, decreased as the number of groups increased (compare the left column in Fig. 1 and the matching off-diagonal panels in Figs. 3 to 6). In other words, while the correlation between estimates was always a negative function of the number of electronic tags and the difference in DM rates between groups, and a positive function of the number tag returns, the magnitude of the correlation decreased with the number of groups involved.

Patterns in the CV of the overall average DM rate with respect to the number of electronic and returned tags varied as a function of the weights applied to each group (Fig. 7). The addition of tag returns did not appreciably reduce the $\mathrm{CV}$ of estimates when group-specific weights were equal, regardless whether there were three or four groups and regardless of different combinations of group-specific DM rates simulated when there were three groups (Fig. 7, top row). However, the addition of tag returns did reduce the $\mathrm{CV}$ when the weight for the group with the smallest DM rate was small, relative to the other groups (Fig. 7, bottom row). The reduction in CV was greatest when the number of electronic and returned tags was small.

\subsection{Case study}

In the case study, DM rate estimation involving only electronic tags was based on a total of 57 haddock for the spring, of which nine died, and 29 for the autumn, of which 13 died (Table 1). The confidence intervals for the two estimates were consequently fairly wide. Based on these data, the survival rate of haddock discarded in the spring was 1.904 (1.279-3.049, 95\% confidence interval) times greater than that for haddock discarded in the autumn.

There were 92 tags returned, of which approximately two-thirds were from fish released in the spring experiment. Based on the number of tag returns relative to releases, survival of haddock discarded in the spring was 1.415 times greater than that for haddock discarded in the fall. This value was within the confidence interval for relative survival based on electronic tagging only, indicating no difference in relative rate between the two types of tags and supporting the assumptions for using the model in eq. 9.

When data for the two types of tags were analysed together, the estimated DM rate for the spring was unchanged and the confidence intervals width was reduced by about $4.8 \%$. The estimate for the autumn was slightly lower than when only electronic tags were considered, and the width of the confidence interval was reduced by about $21.3 \%$.

A new set of simulations was run based on the maximum likelihood parameter estimates and sample sizes in the case study to see if the results of the case study align with predictions from the simulations. From the simulations, the average confidence interval width for estimates including both types of tags divided by the width for estimates based on electronic tags only was 0.884 (0.821-0.946, 95\% interval from the simulations) for the autumn, and $0.972(0.808-1.167)$ for the spring. Thus, the average improvement for the autumn in the simulations (11.6\%) was smaller than in the case study, while for the spring it was similar $(2.8 \%)$. This nonetheless indicates general correspondence between the simulations and the case study, noting that the simulations are 
idealized and that the results for the case study are an inference (i.e., not the true values, as simulated).

\section{Discussion}

Previous studies have jointly analysed electronic tagging and tag-return data to estimate mortality parameters that are difficult to estimate using only one of these methods, such as the joint estimation of natural mortality, DM and fishing mortality (Pollock et al., 2004; Kerns et al., 2016; Hightower and Harris, 2017). However, estimates of different mortality components in these studies were largely, and in some cases exclusively, informed by the data from only one type of tagging. Notably, DM and natural mortality estimates were effectively only informed by the results of the telemetry of electronically tagged fish (e.g., Kerns et al. 2016). We propose a method for an integrated analysis in which information from the two tagging data types inform the estimation of DM rates. We found that the method results in unbiased estimates for which precision can be improved in certain circumstances compared to estimates made using electronic tags alone. Below we discuss the merits of the method, its limitations and how the simulations presented here could be used to plan future DM studies.

When marginal group-specific DM rates are of interest, the proposed model provides more precise estimates than an electronic tagging study alone, and provides absolute rather than the relative estimates, as would be obtained by tag-returns alone. Important improvements in precision with the addition of tag returns were observed for all groups except, in most instances, for the group with the lowest DM rate. Improvements resulting from the addition of traditional tag returns were greatest when the number of electronic tags was small, and the method is therefore particularly suited to studies with small samples as often occurs when pop-up archival tags are used (e.g., Musyl et al. 2014; Musyl and Gilman, 2019). For example, for a study with 50 electronic tags, the CV of estimates for groups other than the one associated with the lowest rate could be reduced by up to $25 \%$ by including between around 40 to 180 tag returns, depending on the number of groups and the difference in DM rates between groups (Figs. 1, 3-6). Similar reductions in CV would require data from approximately an additional 30 to 75 electronic tags in the absence of traditional tag returns. The only simulated exception to the above is the pair comprising groups with rates of 0.30 and 0.25 respectively, where 165 tag returns resulted in a decrease in $\mathrm{CV}$ of about $12.5 \%$ for the first group. Such a reduction could equally be achieved by employing around 20 additional electronic tags.

The addition of tag-return data increased the correlation between group-specific DM rate estimates, thereby limiting the utility of the proposed method in certain circumstance in which the objective is to estimate overall DM rates. Notably when group weights were equal, the inclusion of tag return data provides very little to no improvement in precision. However, as the weight for the group with the smallest DM rate decreases, the precision increases and the gain can be nonnegligible. For example, in simulations for a total of 50 electronic tags, adding 50 tag returns reduced the $\mathrm{CV}$ of the overall DM rate estimate by about $5-25 \%$ (depending on the relative weights and DM rates, and excluding the pair with rates 0.25 and 0.30 ) in the two-group simulations and by about $10-15 \%$ in three and four-group simulations when the weight was small for the group with the smallest DM rate.

It was not surprising that adding tag return data increased the correlation in marginal DM rates between groups, as tag returns inform only on relative survival rates, which are effectively completely correlated between groups. In contrast, group-specific DM rate estimates are uncorrelated when they are based only on electronic tags (Figs. 1, 3-6). Thus, the correlation between group-specific DM rates reflects the contribution of the two types of tags, such that as the 
relative importance of tag returns increases, so does the correlation. While this result is not ideal, our approach nonetheless constitutes an advancement over the existing practice of converting relative DM rate estimates from tag returns to absolute ones using an independently obtained estimate of absolute DM rate for one group (e.g., Kaimmer and Trumble, 1998). That practice results in group-specific rates that are completely correlated and does not provide proper estimates of uncertainty for these rates, unlike our approach.

The results of the simulations can help inform the planning of future DM studies involving the two types of tagging data. In principle, the results can be used to minimise the cost of an experiment for an expected precision, or to maximise the precision for a given budget. However, the optimal design also depends on the tag-return rate, the magnitude of group-specific DM rates and their differences between groups, which in practice typically aren't known a priori. Nonetheless there are a few considerations that could facilitate achieving a design that is at least improved, if not optimal, in practice.

A key consideration is the expense of a properly reporting electronic tag relative to that of a returned traditional tag, as a small reduction in the number of electronic tags could fund a large increase in the deployment and return of traditional tags, thereby facilitating decision making. Electronic tags remain relatively expensive, with acoustic tags such as the ones used in Capizzano et al. (2019) costing around \$650 USD and archival pop-up tags costing several thousand dollars each. Furthermore, these do not reflect the incremental cost per datum as one must also account for factors such as tag failure rates (including incomplete or non-reporting and premature detachment), which can be elevated for pop-up tags (Musyl et al., 2011b), and the likelihood of fish rapidly moving outside of monitoring arrays (for acoustic tags) thereby providing limited or no information on DM. In contrast, the incremental cost of traditional tagging can be negligible if an existing platform for tagging exists (e.g., at-sea observer program) or if a large number of captures to be tagged can be made relatively inexpensively, in which case the major related cost is associated with motivating a reasonable return rate via advertising and incentives (i.e., rewards). This incremental cost could be less that the cost of a single pop-up tag or could be equivalent to forgoing a small number of acoustic tags. While the gains in precision associated with including traditional tag returns may be uncertain prior to the experiment and could be small depending on the circumstances, one must also consider the benefit of tag returns with respect to assessing the potential for experimentally-induced biases in the estimates, a critical consideration when estimating DM rates (ICES, 2014). Specifically, as we argued in the Introduction, the two sources of tagging data together provide an opportunity to asses a possible interaction between electronic tagging induced mortality and group-specific discard mortality if groups are defined based on a pre-release indicator of health. While the method presented here cannot correct for such a bias, identifying it will allow researcher to temper the interpretation of their results.

Another important consideration is the basis for defining the groups of fish for the study. Choosing this basis such that it maximizes differences in the expected group-specific DM rates will also contribute to maximizing the potential gain in precision obtained by including tag returns. Consequently, groups defined using good predictors of survival such as injury or vitality categories (e.g., Benoît et al., 2010; Capizzano et al., 2016; ICES, 2019) or capture and release methods known to induce differential harm (e.g., Benoît et al., 2010; ICES, 2019), are most likely to lead to precision gains. Of course, the basis for defining the groups must not be expected to result in differences in tag return rates following capture, which would violate an assumption of the model or require independent estimation of their value. Consequently, fish size is unlikely to be a suitable basis for defining groups, even if it is an important predictor of DM rates (Davis, 2002; Benoît et 
al., 2013). While there is an advantage to defining the basis for groups at the planning stage, the basis can also be identified during data analysis if significant predictors of mortality are identified post hoc.

Another consideration for experiment planning is the minimum number of electronic tags required for the study. Because the data from these tags are critical to estimating the scale of DM rates, there must be a sufficient number of tags to do so reliably. This is most pertinent when DM rates are expected to be small, which would be associated with a high likelihood of observing no mortality if the number of fish with electronic tags is small. Our simulations provide some predictions in this respect and other experiment-specific scenarios could easily be simulated using the approach employed for this study.

\section{Acknowledgements}

Constructive comments from three anonymous reviewers significantly improved the paper.

\section{References}

Bender, R., Augustin, T., Blettner, M., 2005. Generating survival times to simulate Cox proportional hazard models. Stat. Med. 24, 1713-1723.

Benoît, H.P., Hurlbut, T., Chassé, J., 2010. Assessing the factors influencing discard mortality of demersal fishes in four fisheries using a semi-quantitative indicator of survival potential. Fish. Res. 106, 436-447.

Benoît, H.P., Hurlbut, T., Chassé, J., Jonsen, I.D., 2012. Estimating fishery-scale rates of discard mortality using conditional reasoning. Fish. Res. 125-126, 318-330.

Benoît, H.P., Plante, S., Kroiz, M., Hurlbut, T. 2013., A comparative analysis of marine fish species susceptibilities to discard mortality: effects of environmental factors, individual traits, and phylogeny. ICES J. Mar. Sci. 70, 99-113

Benoît, H.P., Capizzano, C.W., Knotek, R.J., Rudders, R.B., Sulikowski, J.A., Dean, M.J., Hoffman, B., Zemeckis, D.R., Mandelman, J.W., 2015. A generalized model for longitudinal short- and long-term mortality data for commercial fishery discards and recreational fishery catch-and-releases. ICES J. Mar. Sci. 72, 1834-1847.

Campana, S.E., Joyce, W., Manning, M.J., 2009. Bycatch and discard mortality in commercially caught blue sharks Prionace glauca assessed using archival satellite pop-up tags. Mar. Ecol. Prog. Ser. 387, 241-253

Capizzano, C.W., Mandelman, J.W., Hoffman, W.S., Dean, M.J., Zemeckis, D.R. Benoît, H.P., Kneebone, J., Jones, E., Stettner, M.J., Buchan, N.J., Langan, J.A., Sulikowski, J.A., 2016. Estimating and mitigating the discard mortality of Atlantic cod (Gadus morhua) in the Gulf of Maine recreational rod-and-reel fishery. ICES J. Mar. Sci. 73, 2342-2355.

Capizzano, C.W., Zemeckis, D.R., Hoffman, W.S., Benoît, H.P., Jones, E., Dean, M.J., Ribblett, N., Sulikowski, J.A., Mandelman, J.W., (accepted pending revisions). Fishery-scale discard mortality rate estimate for haddock in the Gulf of Maine recreational fishery. N. Am. J. Fish. Manage.

Coggins, L.G.J., Catalano, M.J., Allen, M.S., Pine III, W.E., Walters, C.J., 2007. Effects of cryptic mortality and the hidden costs of using length limits in fishery management. Fish Fish. 8, 196210.

Cook, R.M., 2019. Inclusion of discards in stock assessment models. Fish Fish. 10.1111/faf.12408.

Cooke, S. J., Schramm, H. L., 2007. Catch-and-release science and its application to conservation and management of recreational fisheries. Fish. Manage. Ecol. 14, 73-79. 
Cowx, I. G., Arlinghaus, R., Cooke, S. J., 2010. Harmonizing recreational fisheries and conservation objectives for aquatic biodiversity in inland waters. J. Fish Biol. 76, 2194-2215.

Davies, R.W.D., Cripps, S.J., Nickson, A., Porter, G., 2009. Defining and estimating global marine fisheries bycatch. Mar. Policy 33, 661-672.

Davis, M.W., 2002. Key principles for understanding fish bycatch discard mortality. Can. J. Fish. Aquat. Sci. 59, 1834-1843.

Domeier, M.L., Dewar, H., Nasby-Lucas, N., 2003. Mortality rate of striped marlin (Tetrapturus audax) caught with recreational tackle. Mar. Freshw. Res. 54, 435-445.

European Commission., 2013. European Council Regulation (EU) No 1380/2013 of the European Parliament and of the Council of 11 December 2013 on the Common Fisheries Policy, amending Council Regulations (EC) No 1954/2003 and (EC) No 1224/2009 and repealing Council Regulations (EC) No 2371/2002 and (EC) No 639/2004 and Council Decision 2004/585/EC. Off. J Eur Union L 354, 22-61.

Harrington, J. M., Myers, R. A., Rosenberg, A. A., 2005. Wasted fishery resources: discarded bycatch in the USA. Fish Fish. 6, 350-361.

Hightower, J.E., Harris, J.E., 2017. Estimating fish mortality rates using telemetry and multistate models. Fisheries 42, 210-219.

Hueter, R.E., Manire, C.A., Tyminski, J.P., Hoenig, J.M., Hepworth, D.A., 2006. Assessing mortality of released or discarded fish using a logistic model of relative survival derived from tagging data. Trans. Am. Fish. Soc. 135, 500-508.

ICES. 2014. Report of the Workshop on Methods for Estimating Discard Survival (WKMEDS), 17-21 February 2014, ICES HQ, Copenhagen, Denmark. ICES CM 2014/ACOM:51. 114 pp.

ICES, (2019; In press). ICES WKMEDS Guidelines on methods for estimating discard survival. Breen, M. \& Catchpole, T. (Eds.). ICES Cooperative Research Report.

Ibrahim, J.G., Chen, M.-H., Sinha, D., 2001. Bayesian Survival Analysis. Springer Science+Business Media, Inc. New York.

Kaimmer, S.M., Trumble, R.J., 1998. Injury, condition, and mortality of Pacific halibut bycatch following careful release by Pacific cod and sablefish longline fisheries. Fish. Res. 38, 131-144.

Kerns, J.A., Allen, M.S., Hightower, J.E., 2016. Components of mortality within a black bass highrelease recreational fishery. Trans. Am. Fish. Soc. 145, 578-588.

Kerstetter, D.W., Luckhurst, B.E., Prince, E.D., Graves, J.E., 2003. Use of pop-up satellite archival tags to demonstrate survival of blue marlin (Makaira nigricans) released from pelagic longline gear. Fish. Bull. 101, 939-948.

Maunder, M.N., Punt, A.E., 2013. A review of integrated analysis in fisheries stock assessment. Fish. Res. 142, 61-74.

Musyl, M.K., Gilman, E.L. 2019. Meta-analysis of post-release fishing mortality in apex predatory pelagic sharks and white marlin. Fish Fish. 20, 466-500.

Musyl, M.K., Brill, R.W., Curran, D.S., Fragoso, N.M., McNaughton, L.M., Nielsen, A., Kikkawa, B.S., Moyes, C.D., 2011a. Postrelease survival, vertical and horizontal movements, and thermal habitats of five species of pelagic sharks in the central Pacific Ocean. Fish. Bull. 109, 341-368.

Musyl, M.K., Domeier, M.L., Nasby-Lucas, N., Brill, R.W., McNaughton, L.M., Swimmer, J.Y., Lutcavage, M.S., Wilson, S.G., Galuardi, B., Liddle, J.B. 2011b. Performance of pop-up satellite archival tags. Marine Ecology Progress Series. 433: 1-28.

Musyl, M.K., Moyes, C.D., Brill, R.W., Mourato, B.L., West, A., McNaughton, L.M., Chiang, W.-C., Sun, C.-L., 2014. Postrelease mortality in istiophorid billfish. Can. J. Fish. Aquat. Sci. 72, 538-556. 
Polacheck, T., Eveson, J.P., Laslett, G.M., 2010. Classifying tagging experiments for commercial fisheries into three fundamental types based on design, data requirements and estimable population parameters. Fish Fish. 11, 133-148.

Pollock, K. H., Pine III, W. E., 2007. The design and analysis of field studies to estimate catchand-release mortality. Fish. Manage. Ecol. 14, 1-8.

Pollock, K.H., Jiang, H.H., Hightower, J.E., 2004. Combining telemetry and fisheries tagging models to estimate fishing and natural mortality rates. Trans. Am. Fish. Soc. 133, 639-648.

Punt, A.E., Smith, D.C., Tuck, G.N., Methot, R.D., 2006. Including discard data in fisheries stock assessments: Two case studies from south-eastern Australia. Fish. Res. 79, 239-250.

R Core Team, 2018. R: A language and environment for statistical computing. R Foundation for Statistical Computing, Vienna, Austria. url: http://www.R-project.org.

Raby G. D, Packer J. R, Danylchuk A. J, Cooke S. J., 2014. The understudied and underappreciated role of predation in the mortality of fish released from fishing gears. Fish Fish. 15, 489-505.

Radford, Z., Hyder, K., Zarauz, L.a., Mugerza, E., Ferter, K., Prellezo, R., Strehlow, H.V., Townhill, B., Lewin, W.-C., Weltersbach, M.S., 2018. The impact of marine recreational fishing on key fish stocks in European waters. PLoS ONE 13(9), e0201666.

Stachura, M.M., Lunsford, C.R., Rodgveller, C.J., Heifetz, J., 2012. Estimation of discard mortality of sablefish (Anoplopoma fimbria) in Alaska longline fisheries. Fish. Bull. 110, 271279.

Stansbury, A.L., Götz, T., Deecke, V.B., Janik, V.M. 2015. Grey seals use anthropogenic signals from acoustic tags to locate fish: evidence from a simulated foraging task. Proc. R. Soc. B, 282, 20141595.

Sulikowski, J.A., Benoît, H.P., Capizzano, C.W., Knotek, R.J., Mandelman, J.W., Platz, T., Rudders, D.B., 2018. Evaluating the condition and discard mortality of winter skate, Leucoraja ocellata, following capture and handling in the Atlantic monkfish sink gillnet fishery. Fish. Res. 198, 159-164.

Swain, D.P., Benoît, H.P, Hammill, M.O., Sulikowski, J., 2019. Risk of extinction of a unique skate population due to predation by a recovering marine mammal. Ecol. Applic. 29: e01921

Yergey, M.E., Grothues, T.M., Able, K.W., Crawford, C., DeCristofer, K., 2012. Evaluating discard mortality of summer flounder (Paralichthys dentatus) in the commercial trawl fishery: Developing acoustic telemetry techniques. Fish. Res. 115-116, 72-81

Yochum, N., Stoner, A.W., Sampson, D.B., Rose, C., 2018. A comparison of laboratory-holding and tag return methods for evaluating delayed mortality of Dungeness crab (Cancer magister) discarded in Oregon fisheries. Fish. Bull. 116, 126-141. 
Table 1. Summary of input data sample sizes and analysis results for the case study for the mortality of haddock above the retention size limit as a function of season (from Capizzano et al., accepted). Sample sizes for the electronic tagging data are separated according to the inferred fate of the fish after they were released: died, left-censored and right-censored. Sample sizes for traditionally tagged fish are separated into released (Rel) and returned (Ret) fish. Parameter estimates (with 95\% confidence intervals) are provided for both seasons and for estimation based on an analysis of the electronic tagging data only and with the addition of tag return data.

\begin{tabular}{|c|c|c|c|c|c|c|c|}
\hline \multirow[b]{2}{*}{ Season } & \multicolumn{3}{|c|}{ Electronic } & \multicolumn{2}{|c|}{ Traditional } & \multicolumn{2}{|l|}{ DM rates } \\
\hline & $\begin{array}{l}\text { Die } \\
\mathrm{d}\end{array}$ & $\begin{array}{l}\text { Left } \\
\text { cen. }\end{array}$ & $\begin{array}{l}\text { Right } \\
\text { cen. }\end{array}$ & Rel & Ret & Electronic only & $\begin{array}{l}\text { Electronic } \\
\text { returns }\end{array}$ \\
\hline Spring & 7 & 2 & 48 & 660 & 63 & $0.19(0.11,0.32)$ & $0.19(0.12,0.32)$ \\
\hline Autumn & 12 & 1 & 16 & 430 & 29 & $0.56(0.39,0.72)$ & $0.53(0.39,0.65)$ \\
\hline
\end{tabular}




\section{Figure captions}

Figure 1. Contours, as a function of the number of deployed electronic tags (x-axes; log-scale) and returned traditional tags (y-axes), for the coefficient of variation (CV) of group-specific DM rate estimates for group 1 (left column) and for group 2 (middle column), and contours for the correlation between DM rate estimates for the two groups (right column), as a function of the simulated DM rates (rows, with DM rate values for groups 1 and 2 indicated on the right).

Figure 2. Contours of the coefficient of variation of the overall DM rate estimates, as a function of the number of electronic tags deployed (x-axes; log-scale), returned traditional tags (y-axes), the weights applied to the estimates for the second group (columns; weights indicated above the columns) and the simulated group-specific DM rates (rows, with DM rate values for groups 1 and 2 indicated on the right).

Figure 3. Results for the three-group simulations, with respective group-specific DM rates of 0.75 , 0.70 and 0.5 . Plots on the diagonal: contours, as a function of the number of deployed electronic tags (x-axes; log-scale) and returned traditional tags (y-axes), for the coefficient of variation of group-specific DM rate estimates. Off-diagonal plots: contours of group-pairwise correlation in DM rate estimates.

Figure 4. Results for the three-group simulations, with respective group-specific DM rates of 0.75 , 0.50 and 0.25 . Plots on the diagonal: contours, as a function of the number of deployed electronic tags (x-axes; log-scale) and returned traditional tags (y-axes), for the coefficient of variation of group-specific DM rate estimates. Off-diagonal plots: contours of group-pairwise correlation in DM rate estimates.

Figure 5. Results for the three-group simulations, with respective group-specific DM rates of 0.50, 0.30 and 0.25 . Plots on the diagonal: contours, as a function of the number of deployed electronic tags (x-axes; log-scale) and returned traditional tags (y-axes), for the coefficient of variation of group-specific DM rate estimates. Off-diagonal plots: contours of group-pairwise correlation in DM rate estimates.

Figure 6. Results for the four-group simulations, with respective group-specific DM rates of 0.75 , $0.70,0.50$ and 0.25 . Plots on the diagonal: contours, as a function of the number of deployed electronic tags (x-axes; log-scale) and returned traditional tags (y-axes), for the coefficient of variation of group-specific DM rate estimates. Off-diagonal plots: contours of group-pairwise correlation in DM rate estimates.

Figure 7.Contours of the coefficient of variation of the overall weighted-average DM rate estimates, as a function of the number of electronic tags deployed (x-axes; log-scale) and returned traditional tags (y-axes), for the three three-group simulations and the four-group simulation (columns). The headers above the panels indicate the number of groups and, in the case of analyses based on three groups, the DM rates for the groups. The overall DM rate estimates were derived assuming either equal weights across groups (top row) or assuming a weight of 0.1 for the group with the smallest DM rate and equal weights across remaining groups (bottom row) for illustration purposes. 


\section{Figure 1}

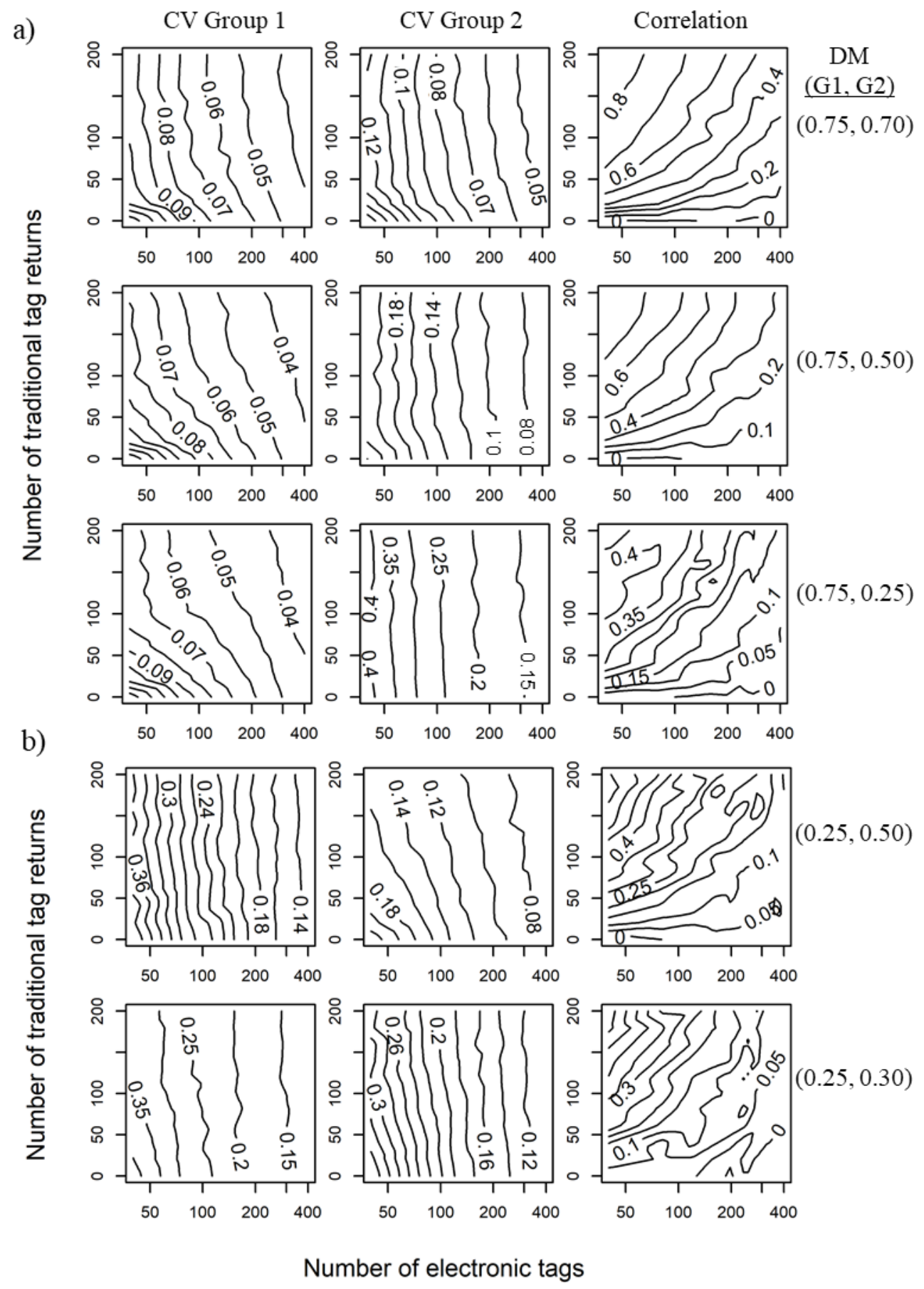


Figure 2

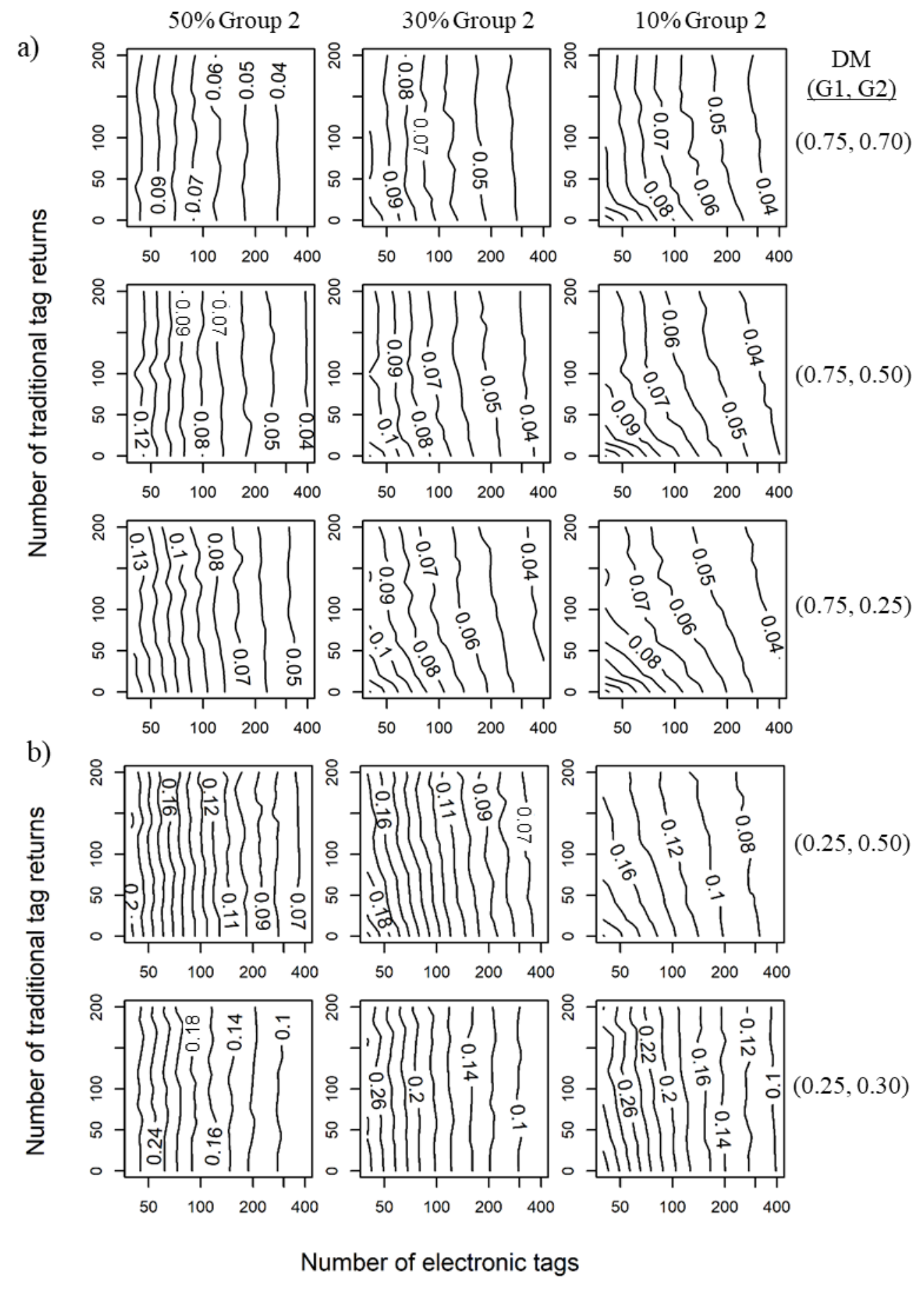

Figure 3 


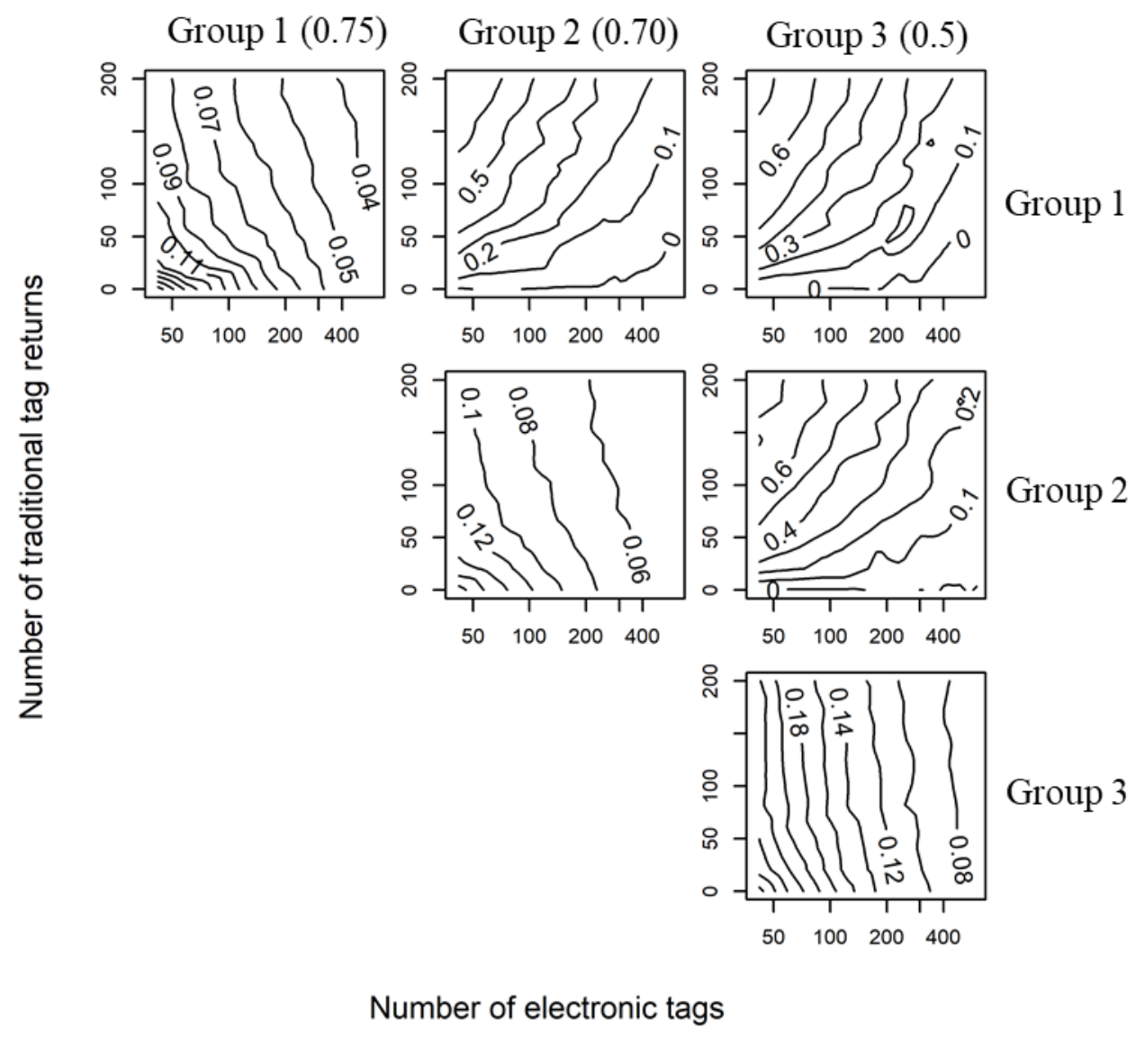




\section{Figure 4}

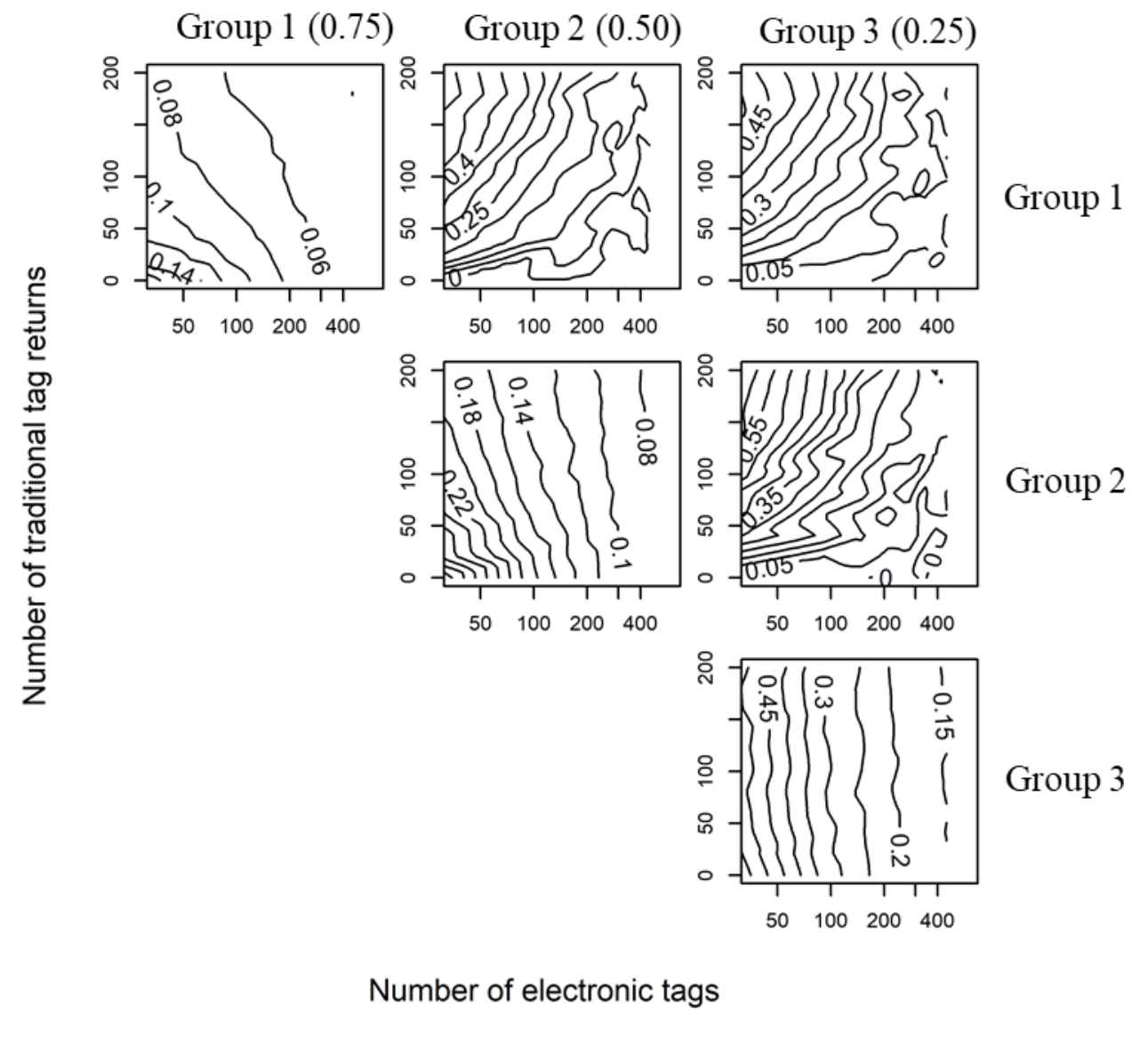




\section{Figure 5}

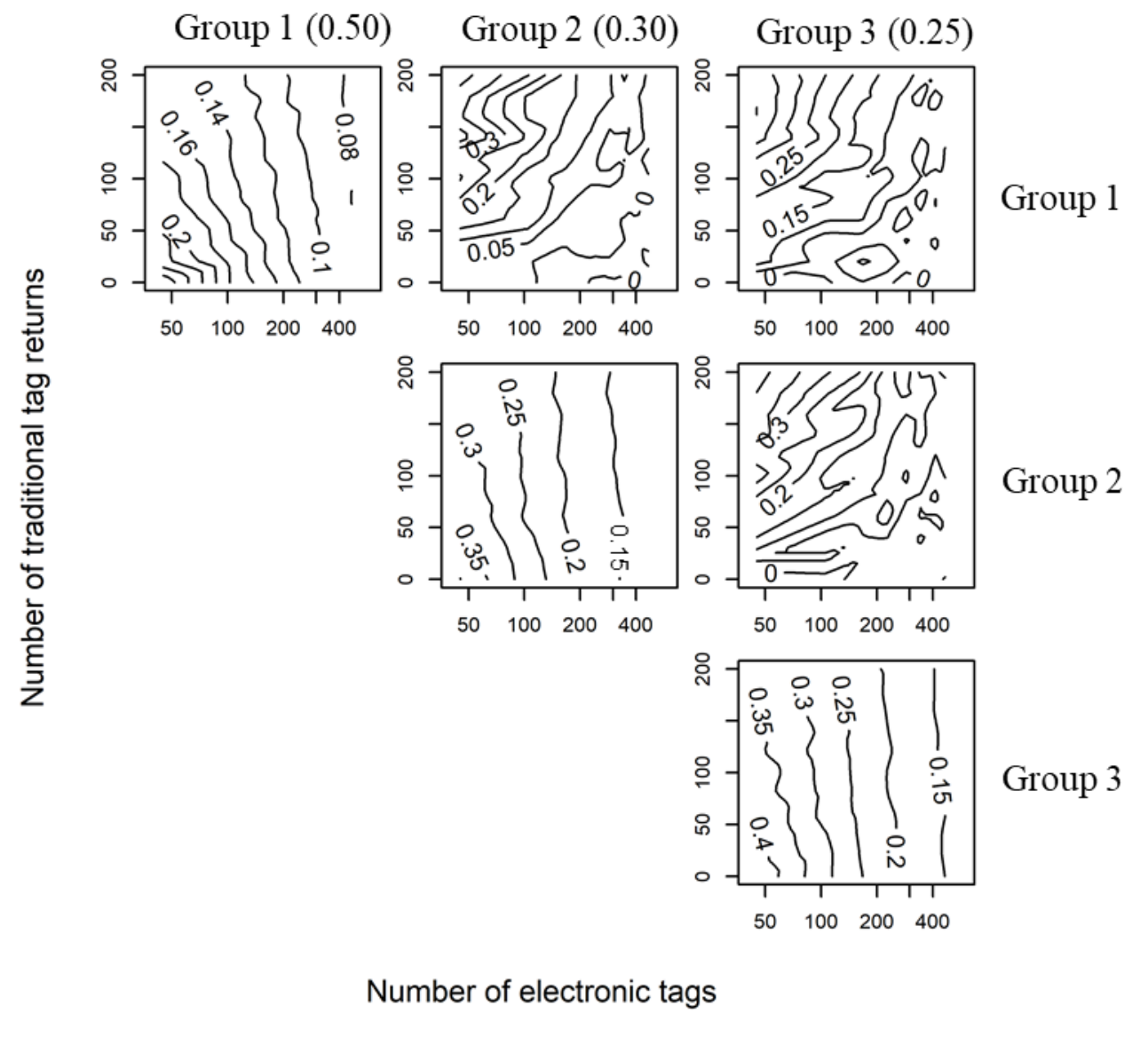




\section{Figure 6}

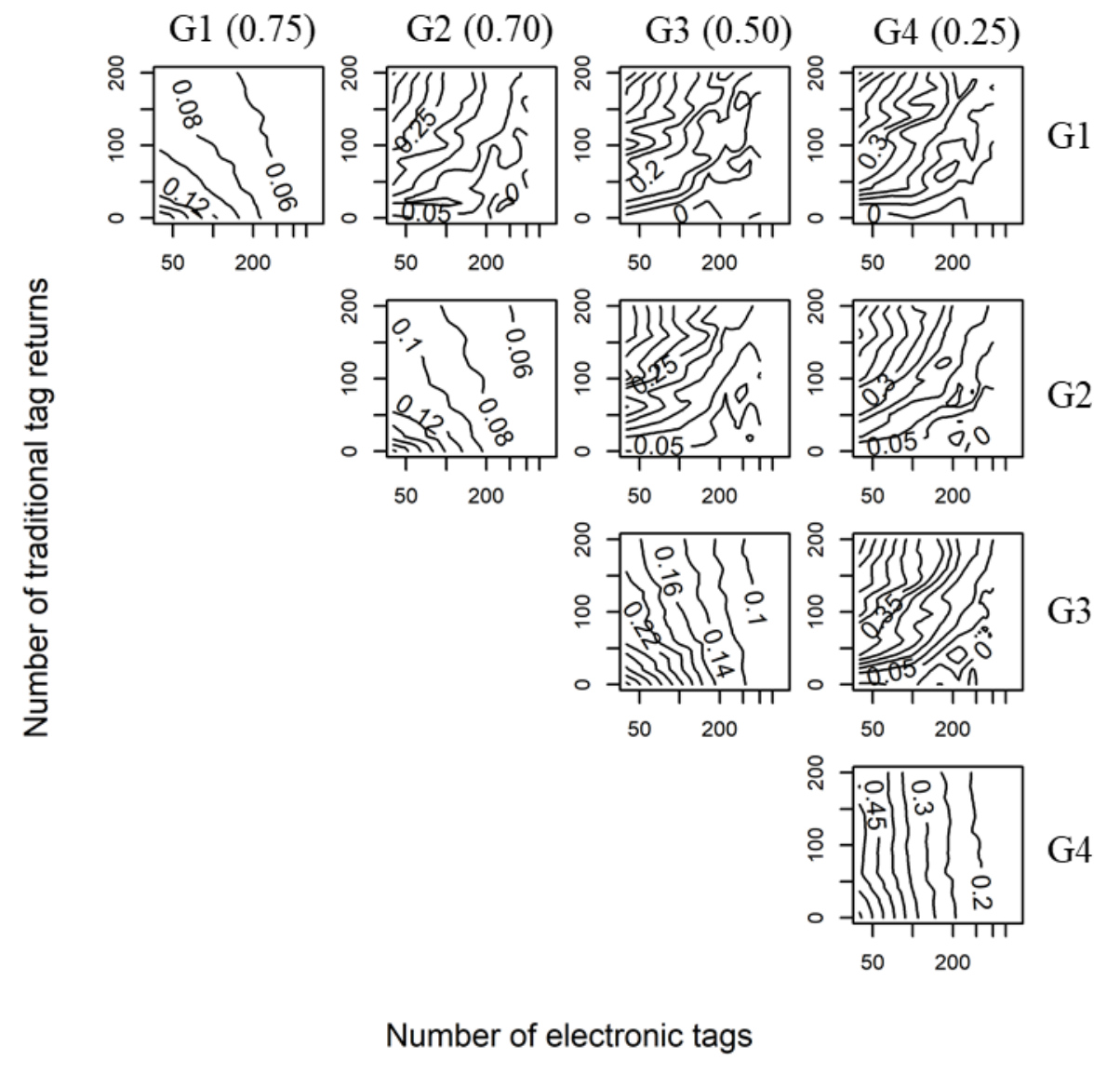


Figure 7

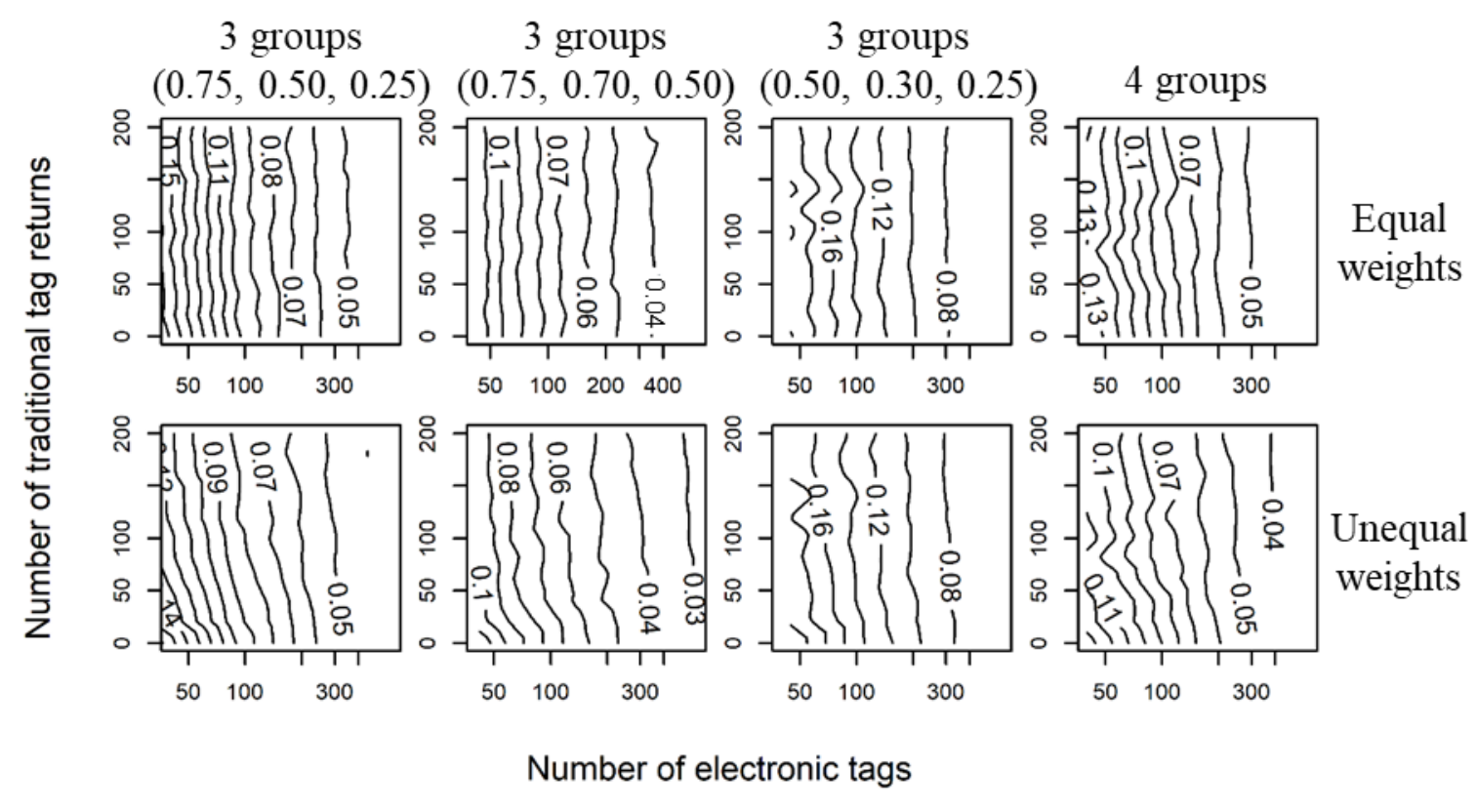

